\title{
Successful treatment of polyarteritis nodosa with intestinal necrosis in a Chinese boy: a case report
}

\author{
Rui Guo ${ }^{1}$, Hongzhen Liu², Xiumei Liu ${ }^{3}$, Wei Liu ${ }^{2}$, Shisong Zhang ${ }^{2}$ \\ ${ }^{1}$ Department of Thoracic and Tumor Surgery, Qilu Children's Hospital of Shandong University, Jinan, China; ${ }^{2}$ Department of General Surgery, Qilu \\ Children's Hospital of Shandong University, Jinan, China; ${ }^{3}$ Department of Pathology, Qilu Children's Hospital of Shandong University, Jinan, China \\ Correspondence to: Shisong Zhang. Department of General Surgery, Qilu Children's Hospital of Shandong University, 23976 Jingshi Road, Huaiyin \\ District, Jinan 250022, China. Email: zsli323@163.com.
}

\begin{abstract}
Polyarteritis nodosa (PAN) is a systemic necrotizing inflammatory disease of the medium and small arteries which has variable clinical manifestations, course, and organ involvement. Intestinal necrosis resulting from PAN is rare, and successful treatment of such cases is even more uncommon. Here, we report the first successful treatment of PAN with intestinal necrosis in a young Chinese child. A 5-year-old boy was admitted to our hospital with a 5-day history of abdominal pain. The patient underwent an emergency exploratory laparotomy, which showed skipping necrosis of the intestinal wall. Intestinal resection and anastomosis failed to improve his abdominal pain. The patient's blood pressure remained high $(140 / 120 \mathrm{mmHg})$, despite captopril treatment. The erythrocyte sedimentation rate (ESR) and D-dimer level were also elevated at $(106.00 \mathrm{~mm} / \mathrm{h}$ and $11.16 \mathrm{mg} / \mathrm{L}$, respectively), as was the 24-hour urine protein $(197.6 \mathrm{mg} / 24 \mathrm{~h})$. Echocardiography revealed that the diameters of the left and right coronary arteries were increased $(0.45$ and $0.49 \mathrm{~cm}$, respectively). Ultrasound showed polyarteritis in the anterior tibial, radial, iliac, and renal arteries. Histopathologic examination revealed elastic fiber rupture and partial mesenteric arteriolar stenosis with occlusion. After 2 months of treatment with systemic methylprednisolone, cyclophosphamide, and prednisone, the patient's abdominal pain was relieved. Furthermore, the patient's ESR and D-dimer levels had reduced to $5 \mathrm{~mm} / \mathrm{h}$ and $0.63 \mathrm{mg} / \mathrm{L}$, respectively; his 24-hour urine protein was normal $(60.0 \mathrm{mg} / 24 \mathrm{~h})$; and his blood pressure had dropped to 101/ $46 \mathrm{mmHg}$. The left and right coronary arteries had reduced to 0.35 and $0.38 \mathrm{~cm}$ in diameter, respectively; however, no significant improvement was observed in the other vessels involved. Unfortunately, the child's parents did not continue to seek medical attention; therefore, his long-term outcome is unknown. In this case, the patient was operated on immediately after symptom onset. The postoperative infection was also quickly controlled, avoiding the occurrence of septic shock. Vascular B-ultrasound and pathology aided in establishing a clear and timely diagnosis, which allowed systematic medical treatment to be delivered, achieving good short-term results.
\end{abstract}

Keywords: Polyarteritis nodosa (PAN); intestinal necrosis; intestinal perforation; case report

Submitted Aug 11, 2021. Accepted for publication Nov 16, 2021.

doi: $10.21037 / \mathrm{tp}-21-378$

View this article at: https://dx.doi.org/10.21037/tp-21-378

^ ORCID: 0000-0001-9683-797X. 


\section{Introduction}

Polyarteritis nodosa (PAN) is a systemic necrotizing inflammatory disease of the medium and small arteries which has variable clinical manifestations, course, and organ involvement. Although all tissues in the body can be involved, the digestive tract is often affected, presenting with mild abdominal pain.

Intestinal necrosis is a common problem in children with acute abdomen, including those with volvulus, internal hernia, intussusception, and incarcerated inguinal hernia. Intestinal necrosis caused by PAN is rare and in its early stages, presents with no obvious intestinal obstruction, which is only the manifestation of acute peritonitis. Therefore, the condition is hidden and can be easily missed by clinicians. Without timely detection, PAN, as a form of systemic segmental vasculitis, can lead to largearea intestinal necrosis and septic shock, which can be lifethreatening in serious cases. Therefore, diagnosis and treatment of children with PAN complicated with intestinal necrosis is challenging, and the mortality rate is high.

To the best of our knowledge, this is the first such report involving a child in China. This case evidences that early surgical and medical treatment of PAN can achieve good short-term outcomes. We present the following case in accordance with the CARE reporting checklist (available at https://tp.amegroups.com/article/view/10.21037/tp-21378/rc).

\section{Case presentation}

A 5-year-old Chinese boy presented at the Qilu Children's Hospital of Shandong University with a 5-day history of episodic abdominal pain with fever $\left(39.0^{\circ} \mathrm{C}\right)$ and convulsions characterized as 1 -minute episodes of limb shaking with clenched teeth. No asphyxia or dyspnea was present. The patient's white blood cell count was elevated to $26.00 \times 10^{9}$ cells/L, which was accompanied by an increase in the percentage of neutrophils ( $85 \%$ ). His C-reactive protein (CRP) level was also elevated $(110.00 \mathrm{mg} / \mathrm{L})$. A CT scan revealed no abnormalities.

An analysis of the patient's cerebrospinal fluid showed reduced chloride $(115.70 \mathrm{mmol} / \mathrm{L})$, glucose elevation $(4.70 \mathrm{mmol} / \mathrm{L})$, and a high cell count $\left(20.00 \times 10^{6} / \mathrm{L}\right)$. Abdominal B-scan ultrasonography showed peritoneal effusion.

Treatment with meropenem, immunoglobulin, and mannitol alleviated the patient's fever and stopped his convulsions; however, this treatment failed to relieve his abdominal pain. After 5 days, the patient presented with angina pectoris, which prompted his referral to the emergency department of our hospital.

During admission, the patient had a normal body temperature $\left(36.8{ }^{\circ} \mathrm{C}\right)$ with high blood pressure $(150 /$ $130 \mathrm{mmHg}$ ) and a poor mental status. Physical examination revealed a "plate-shaped" abdomen with tenderness of the left lower quadrant. No rashes, superficial lymphadenopathies, conjunctival congestion, or abnormal pulsations of the carotid, radial, or femoral arteries were observed. A cardiopulmonary physical examination produced unremarkable results. The patient's extremities were warm with no associated peeling.

Blood tests revealed a normal red blood cell count $\left(4.51 \times 10^{12}\right.$ cells $\left./ \mathrm{L}\right)$, elevated white blood cell $(31.08 \times$ $10^{9}$ cells/L) and platelet $\left(592 \times 10^{9}\right.$ cells $\left./ \mathrm{L}\right)$ counts, and an increased percentage of neutrophils (90\%). The CRP level was high $(171 \mathrm{mg} / \mathrm{L})$. Urinalysis revealed unequivocal urinary protein positivity. The levels of fibrinogen and D-dimer were also elevated $(4.18 \mathrm{~g} / \mathrm{L}$ and $6.02 \mathrm{mg} / \mathrm{L}$, respectively).

Abdominal B-scan ultrasound revealed an abscess in the left abdomen with associated bowel dilatation and effusion, which required an exploratory laparotomy. Intraoperatively, the left lower intestine was discovered to be wrapped by the omentum. There was a small amount of pus discharged after separation. Approximately $45 \mathrm{~cm}$ from the Treitz ligament, $60 \mathrm{~cm}$ of skipping necrosis could be seen in the intestinal wall (Figure 1). After resection of the necrotic intestine, a single-layer end-to-end anastomosis was performed.

After the operation, the patient's temperature, routine blood tests, CRP level, and cerebrospinal fluid findings returned to normal. However, his episodic diffuse abdominal pain persisted. The patient's blood pressure remained high $(140 / 120 \mathrm{mmHg})$, despite captopril treatment. His erythrocyte sedimentation rate (ESR) and D-dimer level were still elevated $(106.00 \mathrm{~mm} / \mathrm{h}$ and $11.16 \mathrm{mg} / \mathrm{L}$, respectively). Abdominal B-scan ultrasound confirmed that there was no anastomotic leakage, intestinal perforation, or ascites, and did not reveal any space-occupying lesion in the adrenal gland. A test for antineutrophil antibodies was negative. MR angiography and venography were normal. Cardiac CT angiography did not show any abnormalities in the aorta or its branches. However, uneven intimal thickening was observed in the anterior tibial, radial, and iliac arteries. The flow velocities of the right and left renal arteries were 44.7 and $42.1 \mathrm{~cm} / \mathrm{s}$, 
respectively, and the resistance indices of the right and left renal arteries were 0.41 and 0.43 , respectively. The left and right coronary arteries measured $0.45 \mathrm{~cm}$ and $0.49 \mathrm{~cm}$ in diameter, respectively. Abdominal contrast-enhanced CT showed low-enhancement areas in both kidneys, which was suggestive of infarction. A urine dipstick test revealed proteinuria, with elevated alpha-1-microglobulin (32.5 mg/L), immunoglobulin G $(9.08 \mathrm{mg} / \mathrm{L})$, and beta-2microglobulin $(>5.95 \mathrm{mg} / \mathrm{L})$, indicating renal tubular and glomerular damage. The 24 -hour urine protein was elevated at $197.6 \mathrm{mg} / 24 \mathrm{~h}$.

The child had a history of fever, high leukocytes, and an

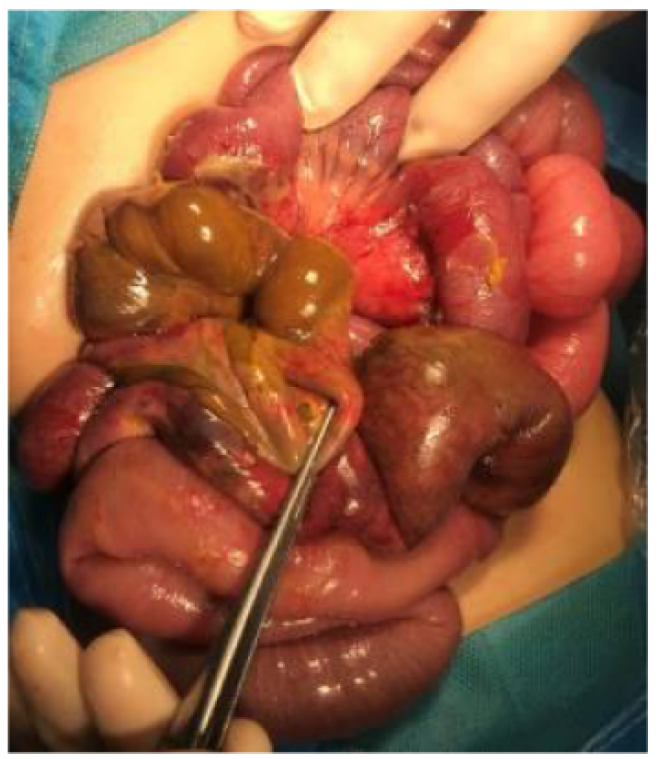

Figure 1 Intraoperative findings. The intestinal wall shows grayish-brown segmental skipping necrosis. elevated ESR. Intestinal necrosis was found during surgery. Postoperative cardiac B-ultrasound examination showed obvious dilation of left and right coronary arteries, which needed to be differentiated from incomplete Kawasaki disease and hemophilia. The child had no transient lymph node enlargement or rash, no peeling on the hands and feet, and normal platelet and coagulation function; incomplete Kawasaki disease was therefore ruled out. Despite the presence of fever, increased leukocytes, and multiple organ damage, there was no splenomegaly or hemocytopenia, fasting triglycerides were normal, and fibrinogen levels were elevated; thus, the possibility of hemophilia could be dismissed.

Histopathologic examination showed that many smalland medium-sized mesenteric arteries had celluloselike necrosis, thrombosis, fibroblastic hyperplasia and hypertrophy, internal and external elastic membrane degeneration and rupture, medial smooth muscle degeneration, lymphocytic and histiocytic infiltration in the intimal and medial smooth muscle tissue, stenosis, and arterial lumen occlusion (Figure 2A-2C). These findings were consistent with a diagnosis of PAN, despite the patient having no family history of this disease.

After 2 months of treatment with systemic methylprednisolone, cyclophosphamide, and prednisone, the patient's abdominal pain was relieved. His ESR and D-dimer levels had reduced to $5 \mathrm{~mm} / \mathrm{h}$ and $0.63 \mathrm{mg} / \mathrm{L}$, respectively, and he had normal 24-hour urine protein $(60.0 \mathrm{mg} / 24 \mathrm{~h})$. Urine dipstick urinalysis showed proteinuria, with decreased alpha-1-microglobulin, immunoglobulin $G$, and beta2 -microglobulin (22.5, 7.9, and 1.87, respectively). The patient's blood pressure had decreased to $101 / 46 \mathrm{mmHg}$. The diameters of the left and right coronary arteries had
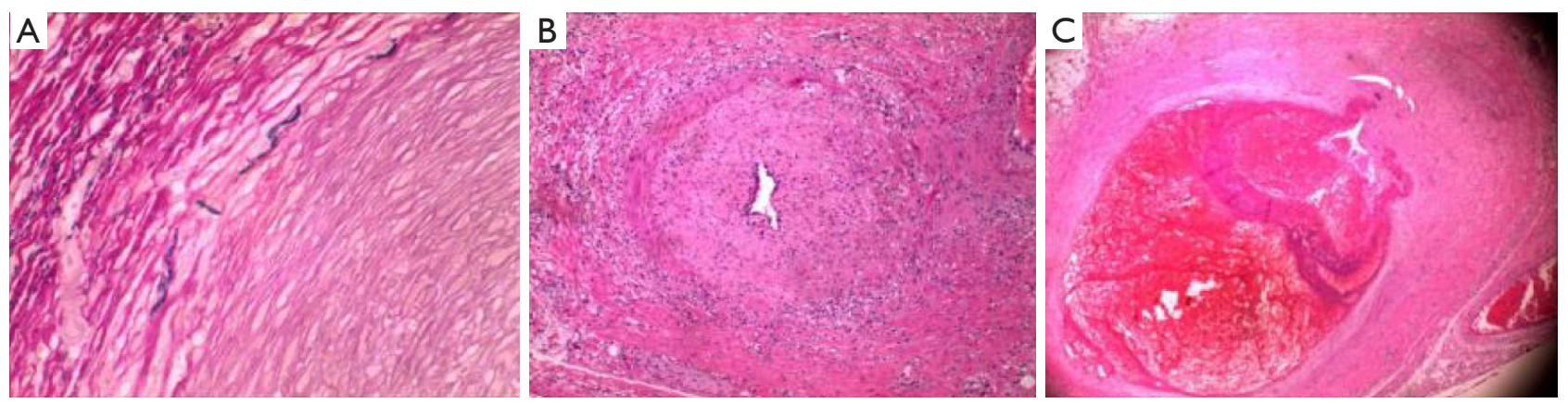

Figure 2 Histopathologic findings. (A) The elastic fibers of the mesenteric arterioles are shown to be ruptured (elastin and Van Gieson stain, $\times 10$ ); (B) mesenteric arteriole stenosis (hematoxylin and eosin stain, $\times 20$ ); (C) mesenteric arteriole occlusion (hematoxylin and eosin stain, $\times 40)$. 


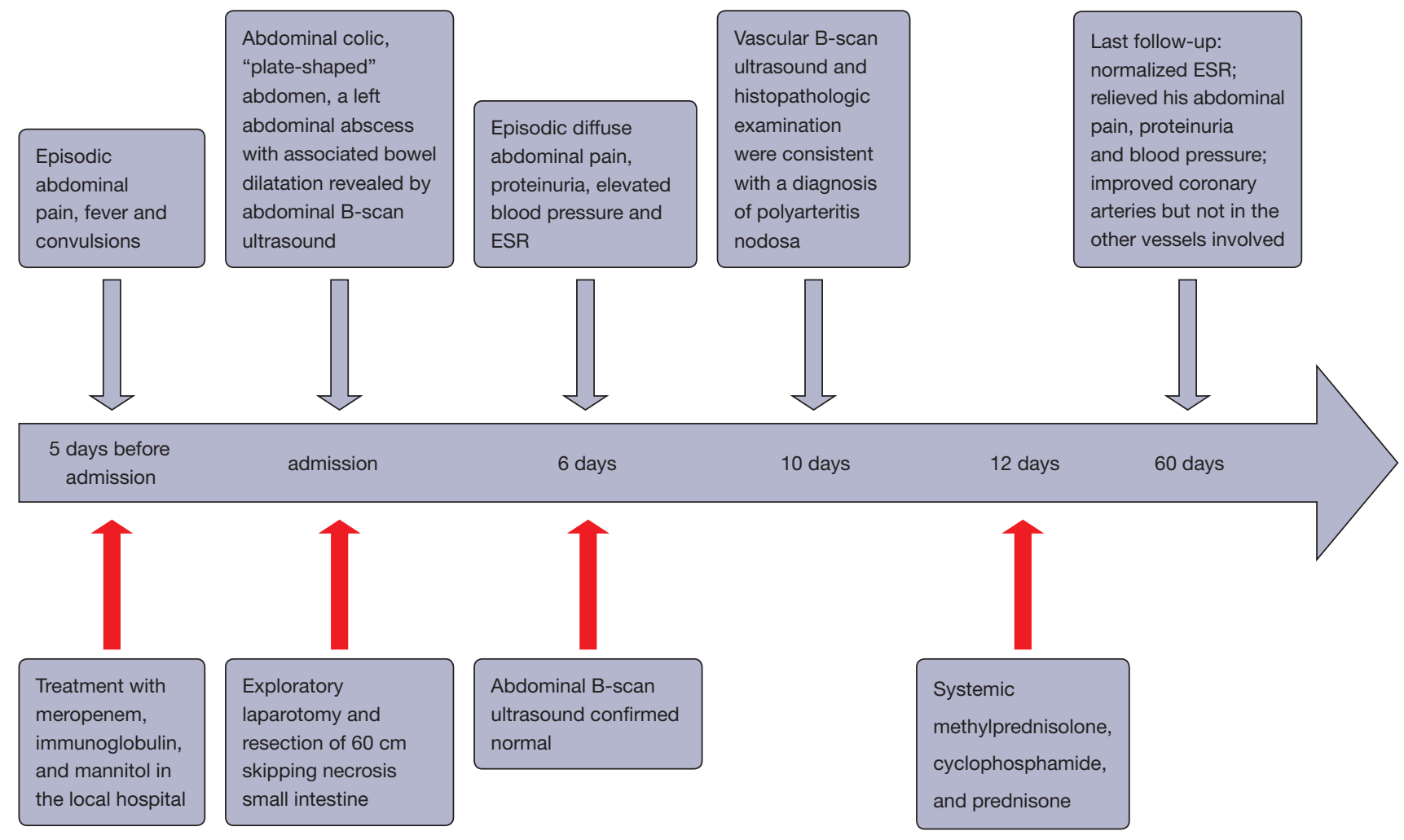

Figure 3 Timeline of this case. ESR, erythrocyte sedimentation rate.

narrowed to 0.35 and $0.38 \mathrm{~cm}$, respectively; however, no significant improvement was seen in the other vessels involved (Figure 3).

These outcomes were encouraging, which led to the continuation of treatment with systemic methylprednisolone, cyclophosphamide, and prednisone.

Unfortunately, the child's parents did not continue to seek medical attention; therefore, his long-term outcome is unknown.

All procedures performed in studies involving human participants were in accordance with the ethical standards of the institutional and/or national research committee(s) and with the Helsinki Declaration (as revised in 2013). Written informed consent was obtained from the patient's parents for publication of this case report and accompanying images. A copy of the written consent is available for review by the editorial office of this journal.

\section{Discussion}

Intestinal necrosis is a common condition in children with acute abdomen. Intestinal necrosis caused by PAN is rare, and successful treatment of such cases is even more uncommon. To the best of our knowledge, this is the first report of successful treatment of PAN in a child in China.

PAN is a type of systemic segmental vasculitis which mainly involves small- and medium-sized muscular arteries. The incidence of PAN in adults is known to be $0.002 \%$ to $0.009 \%$, but there are no data regarding its incidence in children (1). Cases of PAN vary in terms of clinical manifestations, disease course, and organ involvement. Although PAN may affect any organ in the body, data show that peripheral nerves (79\%) and the kidneys (66.2\%) are the most vulnerable body parts, followed by the skin (49.7\%), gastrointestinal tract (35.6\%), and heart (34.8\%) (2). In the above-described case, multiple organs (the intestine, kidney, and heart) were involved simultaneously.

Digestive tract involvement is common in PAN, and mainly manifests with mild abdominal pain. Intestinal necrosis is a rare manifestation of PAN in children and usually indicates a poor prognosis (3), as multiple organs are often involved by the time it occurs. Failure to manage the disease in time can result in death.

Through a literature search, we only found eight 
previous cases of PAN in children, including four cases who died (two cases died due to multiple organ failure and two cases died from septic shock caused by intestinal perforation) (4-9). In our case, surgery was performed immediately after symptom onset, at which point intestinal perforation due to segmental necrosis had not yet occurred. After intestinal resection and anastomosis, the patient's blood parameters and CRP level normalized. Owing to this case of PAN being detected at an early stage, appropriate medical and surgical management could be performed promptly, resulting in a good short-term clinical outcome.

To date, an optimal evidence-based treatment for PAN has yet to be established. The main considerations for the management of PAN are the degree of organ involvement and disease progression. The French Vasculitis Group (FVSG) proposed the Five-Factor Score (FFS) for evaluating the disease severity and prognosis of systemic necrotizing vasculitis and guiding clinical treatment (10). The five factors include severe gastrointestinal disease (defined as bleeding, obstruction, perforation, or pancreatitis), renal insufficiency (defined as serum creatinine $>140 \mu \mathrm{mol} / \mathrm{L}$ ), proteinuria $\geq 1 \mathrm{~g} / 24 \mathrm{~h}$, heart disease (defined as infarction or heart failure), and central nervous system involvement. When important organs are involved (i.e., FFS $\geq 1$ ), combination therapy with immunosuppressive agents and corticosteroids are given. Cyclophosphamide is the preferred immunosuppressant for patients with PAN who have rapid progression or important organ involvement (11). In our case, intestinal necrosis occurred with renal and cardiac involvement (i.e., FFS $\geq 1$ ), which prompted treatment with methylprednisolone, cyclophosphamide, and prednisone. After treatment, the patient's elevated blood pressure and ESR, and proteinuria were resolved. A significant decrease was also seen in the degree of coronary artery dilation, although the other involved vessels showed no similar improvement. The long-term prognosis of our patient is unknown, but our short-term observations suggest that the treatment was effective.

In conclusion, PAN with intestinal necrosis in children is extremely rare and has a high mortality rate. In this case, surgery was performed immediately after symptom onset, and the patient's postoperative infection was quickly controlled, avoiding the occurrence of septic shock. Owing to a lack of understanding of this disease, we were perplexed when the patient experienced uncontrollable abdominal pain and hypertension after the surgery. Fortunately, vascular B-ultrasound and pathology helped us to make a clear and timely diagnosis, which allowed for systematic medical treatment to be delivered, achieving good shortterm results. Recovery from vascular inflammation determines the long-term prognosis of patients with PAN, and requires systematic medical treatment and long-term follow-up. Unfortunately, after 2 months of treatment, our patient did not continue to seek medical attention; therefore, the long-term benefits of systematic treatment and the extent of recovery of the involved blood vessels are unknown.

\section{Acknowledgments}

We thank B. Meiser and J. Reynolds for its linguistic assistance during the preparation of this manuscript.

Funding: None.

\section{Footnote}

Reporting Checklist: The authors have completed the CARE reporting checklist. Available at https://tp.amegroups.com/ article/view/10.21037/tp-21-378/rc

Conflicts of Interest: All authors have completed the ICMJE uniform disclosure form (available at https://tp.amegroups. com/article/view/10.21037/tp-21-378/coif). The authors have no conflicts of interest to declare.

Ethical Statement: The authors are accountable for all aspects of the work in ensuring that questions related to the accuracy or integrity of any part of the work are appropriately investigated and resolved. All procedures performed in studies involving human participants were in accordance with the ethical standards of the institutional and/or national research committee(s) and with the Helsinki Declaration (as revised in 2013). Written informed consent was obtained from the patient's parents for publication of this case report and accompanying images. A copy of the written consent is available for review by the editorial office of this journal.

Open Access Statement: This is an Open Access article distributed in accordance with the Creative Commons Attribution-NonCommercial-NoDerivs 4.0 International License (CC BY-NC-ND 4.0), which permits the noncommercial replication and distribution of the article with the strict proviso that no changes or edits are made and the original work is properly cited (including links to both the 
formal publication through the relevant DOI and the license). See: https://creativecommons.org/licenses/by-nc-nd/4.0/.

\section{References}

1. Karadag O, Erden A, Bilginer Y, et al. A retrospective study comparing the phenotype and outcomes of patients with polyarteritis nodosa between UK and Turkish cohorts. Rheumatol Int 2018;38:1833-40.

2. Pagnoux C, Seror R, Henegar C, et al. Clinical features and outcomes in 348 patients with polyarteritis nodosa: a systematic retrospective study of patients diagnosed between 1963 and 2005 and entered into the French Vasculitis Study Group Database. Arthritis Rheum 2010;62:616-26.

3. Yajima S, Asano H, Fukano H, et al. Necrosis of the small intestine leading to a diagnosis of polyarteritis nodosa: a case report. J Med Case Rep 2019;13:55.

4. Oğuzkurt P, Senocak ME, Ciftci AO, et al. Mesenteric vascular occlusion resulting in intestinal necrosis in children. J Pediatr Surg 2000;35:1161-4.

5. Gündoğdu HZ, Kale G, Tanyel FC, et al. Intestinal

Cite this article as: Guo R, Liu H, Liu X, Liu W, Zhang S. Successful treatment of polyarteritis nodosa with intestinal necrosis in a Chinese boy: a case report. Transl Pediatr 2022;11(1):157-162. doi: 10.21037/tp-21-378 perforation as an initial presentation of polyarteritis nodosa in an 8-year-old boy. J Pediatr Surg 1993;28:632-4.

6. Chattopadhyay A. Intestinal perforation due to polyarteritis nodosa. Indian J Pediatr 2001;68:297-8.

7. de Carpi JM, Castejón E, Masiques L, et al. Gastrointestinal involvement in pediatric polyarteritis nodosa. J Pediatr Gastroenterol Nutr 2007;44:274-8.

8. Venuta A, Ceccarelli PL, Biondini D, et al. Jejunal obstruction as initial presentation of polyarteritis nodosa in a 13-month-old boy. J Pediatr Surg 2011;46:E27-9.

9. Gomes RC, Marques VL, Cavalcante EG, et al. Severe intestinal involvement as initial manifestation of systemic childhood polyarteritis nodosa: report of two cases. J Pediatr Surg 2013;48:425-8.

10. Erden A, Batu ED, Sönmez HE, et al. Comparing polyarteritis nodosa in children and adults: a single center study. Int J Rheum Dis 2017;20:1016-22.

11. De Virgilio A, Greco A, Magliulo G, et al. Polyarteritis nodosa: A contemporary overview. Autoimmun Rev 2016;15:564-70. 